\title{
MicroRNA-31 Can Positively Regulate the Proliferation, Differentiation and Migration of Keratinocytes
}

\author{
Fei Wang ${ }^{a}$ Yuantao Gao ${ }^{b}$ Yitong Yuan ${ }^{a}$ Ruochen Du ${ }^{a}$ Pengfei Li ${ }^{a}$ \\ Fang Liu $^{a}$ Ye Tian $^{a}$ Yali Wang $^{a}$ Ruxin Zhang ${ }^{a}$ Bichun Zhao $^{a}$ \\ Chunfang Wang ${ }^{a}$ \\ aLaboratory Animal Center, Shanxi Medical University, Taiyuan, China; \\ ${ }^{b}$ Nanchang University Queen Mary School, Nanchang, China
}

\section{What Is It about?}

This review summarized the regulation roles of miR-31 in keratinocytes via regulating the NF- $\kappa \mathrm{B}$, RAS/MAPK, and Notch signaling pathways. The positive feedback loop formed by miR-31 and the NF- $\kappa B$ signaling pathway in keratinocytes was focused on, which will play an important role in preventing and treating psoriasis. In addition, given the positive regulatory effect of miR-31 in keratinocytes, miR-31 has the potential therapeutic value for targeted treatment of various keratinocyterelated skin diseases.

\section{Keywords}

Keratinocytes $\cdot$ MicroRNAs $\cdot$ MicroRNA-31 $\cdot$ NF- KB signaling pathways

\begin{abstract}
In the past decades, the key roles of most microRNA in dermatosis and skin development have been explored one after another. Among them, microRNA-31 (miR-31) has a prominent role in the regulation of keratinocytes. Numerous studies show that miR-31 can positively regulate the proliferation, differentiation and cell activity of keratinocytes via regulating the NF- $\mathrm{kB}$, RAS/MAPK, Notch signaling pathways, and some cytokines. At present, the interaction between miR-31 and the NF-KB signaling pathway in keratinocytes is a hot research topic. The positive feedback loop formed by miR-31 and NF-KB signaling may bring new ideas for the prevention of psoriasis. The abnormal state of keratinocytes is usually the pathological basis of many skin and immune system diseases. Therefore, strengthening the ability to regulate keratinocytes may be a breakthrough for a variety of diseases. At the same time, miR-31's capacity to accelerate wound healing via positively regulating keratinocytes should be further investigated in the treatment of chronic ulcers and trauma.


Wang et al.: Important Regulator of Keratinocytes: miR-31

\section{Introduction}

Skin, composed of the epidermis and the underlying dermis, is the largest organ in the body and fulfils a variety of functions. Keratinocytes, the principle epidermal cells, are continuously renewed by the proliferation of stem cells and the differentiation of their progeny, and also directly or indirectly participate in many physiological and pathological processes [1]. For instance, the cutaneous wound healing process requires proper migration and proliferation of keratinocytes at the periphery of the wound to resurface the skin wound with new epithelium [2]. Proliferation inhibition of keratinocytes may result in defects of re-epithelialization, which is associated with chronic non-healing wounds [3]. In addition, abnormal proliferation and differentiation of keratinocytes can lead to skin diseases such as psoriasis, allergic contact dermatitis, acanthosis nigricans, and pemphigus [4-7]. Gröne et al. [8] concluded that keratinocytes can produce many of the currently identified cytokines which include interleukin (IL)-1, -6, -7, -8, -10, 12, -15, -18, and -20, tumor necrosis factor alpha (TNF- $\alpha$ ), and interferon- $\alpha,-\beta$, and $-\gamma$, in both physiological state or stress state. Meanwhile, IL-4, IL-13, IL-17, and IL-2 receptors have also been found in keratinocytes. In addition, IL-21, IL-23, and IL-36 $\gamma$ have been shown to be associated with abnormal proliferation of keratinocytes [9-11]. Given the close relationship between keratinocytes and various cytokines, the combination should receive more attention from both immunologists and dermatopathologists.

The first confirmed microRNAs (miRNAs) were lin-4 and let-7, which were found in nematode worms in 1993 through the characteristics of the $C$. elegans heterochronic gene lin-4 [12]. miRNA is a type of non-coding single-stranded RNA molecule encoded by endogenous genes with a length of about 22 nucleotides [13]. According to current research, to date nearly 2,500 human miRNAs have been identified and as novel regulators of gene expression participate in almost all cellular procedures, such as development, organogenesis, apoptosis, cell proliferation, and other biological functions $[14,15]$. Results from numerous studies have indicated that a substantial number of miRNAs (Table 1) have been identified in skin and play critical roles as a class of posttranscriptional gene regulator factors in skin development and skin diseases [16].

We focus on microRNA-31 (miR-31) in this review, the biogenesis of which is quite complex as in other miRNAs. Two intermediate forms of miR-31, primary (pri-) and precursor (pre-) miR-31, is produced sequentially via the effect of RNA polymerase (RNAP) II and a microprocessor complex during the maturation process. The pre-miR-31 is carried from the nucleus to the cytoplasm via exportin 5 (XPO5), and then cleaved by the endonuclease Dicer into miR-31 duplex in the cytoplasm. miR-31 can bind to an argonaute (Ago) protein to form a mature RNA-induced silencing complex (RISC), which is then guided to the $3^{\prime}$-untranslated region ( $3^{\prime}$-UTR) of the target mRNA to exert its regulatory function by binding to target transcripts in most cases (Fig. 1) [40-42].

\section{Why Is miR-31 Important Regulator of Keratinocytes?}

Notably, the relationship between miR-31 and various skin diseases has been increasingly studied in recent years (Table 1). In view of the current and previous studies, we suggest that the mechanism of action is predominantly concerned with the regulating the effect of keratinocytes by miR-31. Using microarray analysis, Chen et al. [43] provided the first detailed molecular profile of the dynamic time course of the transcriptome and microRNAome in oral mucosa and skin wound healing models. They subsequently examined the differentially and highly expressed microRNAome on oral mucosa and skin epithelium, 


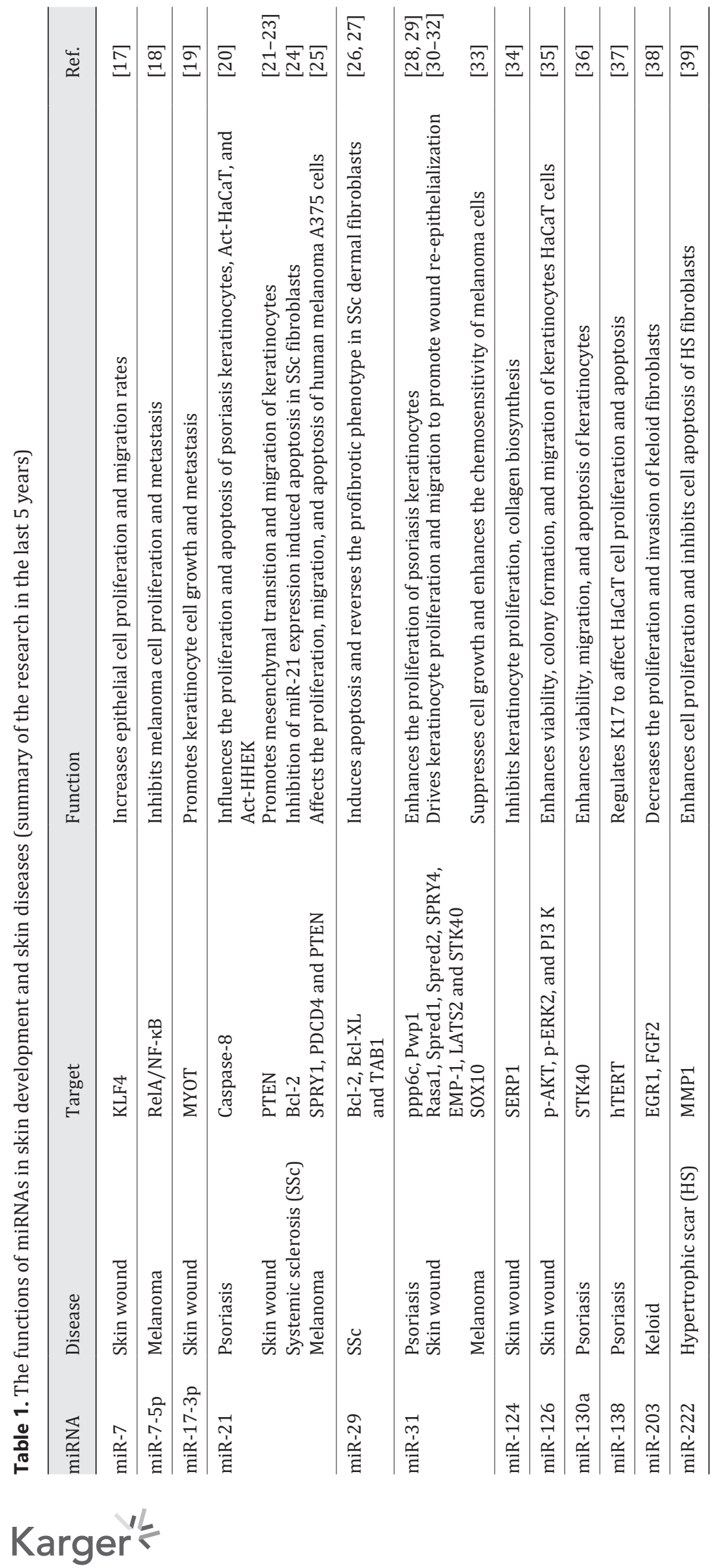




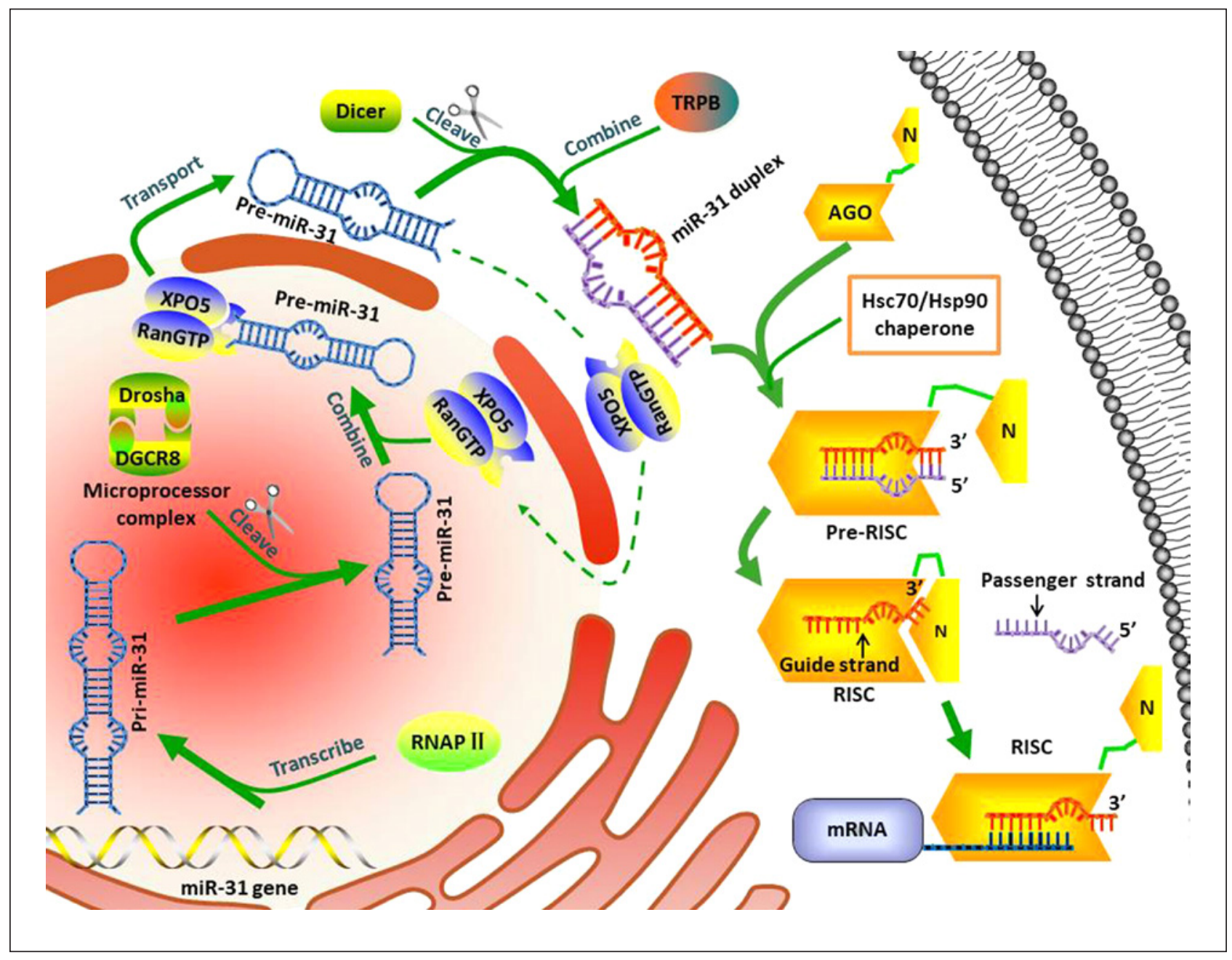

Fig. 1. The biogenesis of miR-31. First of all, the miR-31 gene is transcribed, predominantly by RNAP II, into pri-miR-31, which is cleaved by a microprocessor complex comprised of the DiGeorge syndrome chromosomal region 8 (DGCR8) and the RNase III enzyme Drosha into pre-miR-31 with a 70-nt stem-loop structure. Secondly, the Pre-miR-31 is exported from the nucleus to the cytoplasm by a complex consisting of XPO5 and GTP-binding nuclear protein Ran (RanGTP). In the cytoplasm, Pre-miR-31 is cleaved from the terminal loop by the RNase III endonuclease Dicer with the cooperation of the transactivating response RNA binding protein (TRBP) to form miR-31 duplex. Thirdly, miR-31 duplex (the guide strand and the passenger strand are shown in red and purple, respectively) is loaded into the AGO protein through the Hsc70/Hsp90 molecular chaperone mechanism to form a complex called pre-RISC. The maturation of RISC is completed by passenger strand ejection, in which the $\mathrm{N}$ domain of AGO proteins pries open base pairs at the $3^{\prime}$ end of the guide strand and then the passenger strand is ejected from AGO proteins. Finally, in most cases, the mature RISC induces translational inhibition of the specific mRNA target.

which were then grouped into 5 clusters based on their expression patterns. Cluster IV, consisting of 8 miRNAs, including miR-31, showed the most tissue specificity $(66.7 \%)$. miR-31 exhibited the most consistency between miR-Seq and LCM/TaqMan analysis, whether in comparison with the 8 miRNAs of Cluster IV or the selected miRNAs of other clusters [44, 45]. Surprisingly, Chen et al. [45] confirmed that miR-31 is superior to miR-21 (a recognized enhancer of wound healing) resulting in accelerated wound closure in vitro wound-healing assay. The result of the in vivo wound-healing assay was the same as that of 
the in vitro assay. Those studies suggested that the differentially and highly expressed microRNAome may underlie the capacity that wounds within the oral mucosa can heal more rapidly than skin wound. miR-31 is the highest and most stable expression among microRNAome in keratinocytes, whether in oral mucosa or skin wound healing models. And the effects of miR-31 on wound closure are more effective than other miRNAs. However, Chen et al. [45] have not mentioned the mechanism of miR-31 in regulating keratinocytes on oral mucosa wound healing. Therefore, we do not know whether the role of miR-31 in promoting oral mucosa wound healing is achieved through the currently proven regulatory pathways and target genes.

\section{How Does miR-31 Regulate Keratinocytes?}

The biogenesis of miR-31 clearly shows that miR-31 will combine with the specific mRNA to regulate the expression of specific proteins and activate the specific signal pathways, thereby completing biological regulatory functions [46]. Therefore, based on the existing results of experiments, we summarize which proteins and signaling pathways miR-31 can use to regulate the proliferation and migration of keratinocytes.

\section{Nuclear Factor-kB Signaling Pathway}

$\mathrm{Xu}$ et al. [49] examined the expression of miR-31 in psoriatic keratinocytes. The typical characteristics of the lesion are mainly hyperproliferation and abnormal differentiation of keratinocytes, according to the distinct miRNA expression profile in psoriasis skin compared with healthy skin $[47,48]$, and the results demonstrated that miR-31 is markedly overexpressed in keratinocytes of psoriasis skin lesions. Above all, they demonstrated that inhibition of the expression of endogenous miR-31 efficiently suppressed the production of these cytokines/chemokines (including IL-1b, CXCL1, CXCL5, and CXCL8/IL-8) in keratinocytes which were treated with the proinflammatory cytokine TNF- $\alpha$. Subsequently, the nuclear factor- $\kappa \mathrm{B}(\mathrm{NF}-\kappa \mathrm{B})$ signaling pathway, one of the signaling pathways regulating the expression of IL-1b, CXCL1, CXCL5, and CXCL8/IL-8, was proved to be inhibited when endogenous miR-31 was suppressed. In the NF- $\kappa B$ signaling pathway, STK40, a negative regulator of the NF- $\kappa B$ signaling pathway, was proved to be a direct target gene for miR-31 by connecting to the two binding sites in its 39-UTR. Finally, they demonstrated that TGF- $\beta 1$, a cytokine highly expressed in psoriasis epidermis, upregulated miR-31 expression in keratinocytes in vitro and in vivo [49]. This study preliminarily established the regulation process that miR-31 modified the NF- $\kappa$ B signaling pathway in keratinocytes of psoriatic lesions. They indicated that miR-31 activates the NF- $\kappa B$ signaling pathway by inhibiting STK40, which results in some cytokines/chemokines being secreted by keratinocytes to attract immune cells to migrate into epidermis and dermis from vessels, while immune cell-derived cytokines in turn act on keratinocytes to promote keratinocyte proliferation and inhibit keratinocyte differentiation.

As a protein transcription factor, NF- $\mathrm{\kappa B}$ can regulate the activity of a large number of proinflammatory genes and it is considered to be a key mediator involved in the pathogenesis of psoriasis [50]. Yan et al. [28] revealed the mechanism that activated NF- $\kappa B$ can promote keratinocyte hyperproliferation in psoriasis in via inducing miR-31. Firstly, miR-31 was the most highly expressed miRNA in psoriasis mice models. Moreover, they performed chromatin immunoprecipitation assays to confirm that IL- 6 stimulation caused $\mathrm{p} 65$ to recruit to specific mir-31 promoter leading to miR-31 upregulation. Finally, to identify the direct target of 


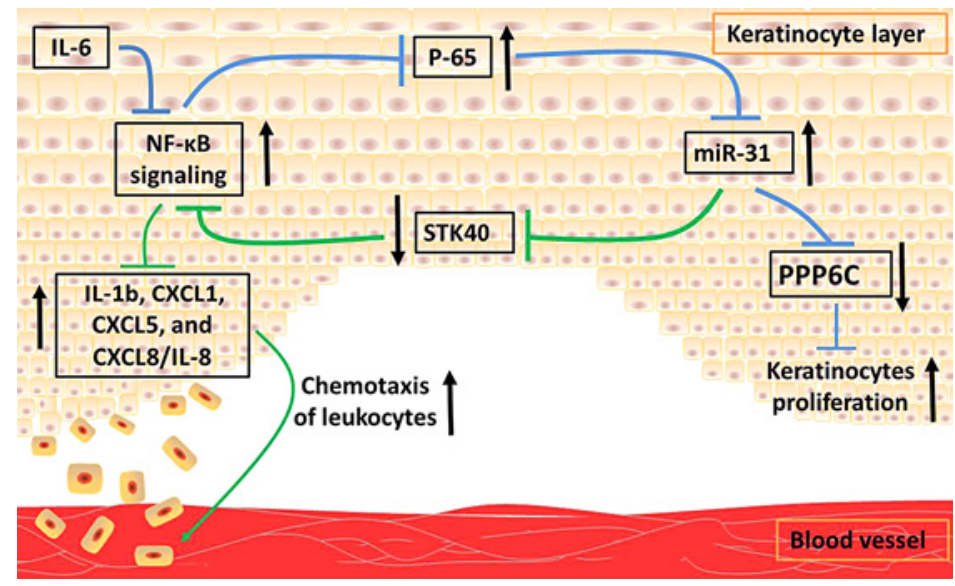

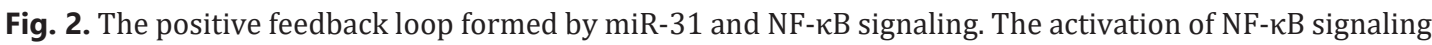
induced by IL- 6 can upregulate the expression of P-65 and further promote the expression of miR-31. The high expression of miR-31 can downregulate the expression of PPP6C, a negative regulator of keratinocyte proliferation, thereby promoting the proliferation of keratinocytes. In addition, high expression of miR-31 can also inhibit STK40, a negative regulator of NF- $\mathrm{B}$ signaling. The expression of IL-1b, CXCL1, CXCL5, and CXCL8/IL-8 will be upregulated by activated NF- $\kappa$ B signaling. These cytokines contribute to the chemotaxis of leukocytes, allowing leukocytes to migrate from blood vessels into skin and accelerate the proliferation of keratinocytes.

miR-31 in the regulation of keratinocytes, Yan et al. [28] identified 20 potential target genes. Among them, as a negative regulator of the cell cycle, protein phosphatase 6 (ppp6c), which is diminished in the human psoriasis epidermis, has been noted. And then, the results demonstrated that miR-31 could directly target the sequence in the 30-UTR of ppp6c mRNA to induce ppp6c silencing which led to increased epidermal thickness and increased keratinocyte proliferation. Together, these experiments suggest that NF- $\kappa B$ signaling activation triggered by inflammatory cytokines upregulates miR-31, which represses ppp6c. The downregulation of ppp6c exacerbates the excessive proliferation and epidermal proliferation of keratinocytes [28]. This study reveals another action pathway between miR-31 and NF- $\kappa B$ signaling in promoting keratinocyte proliferation: activated NF- $\kappa B$ signaling can induce the high expression of miR-31. Subsequently, another team also demonstrated that the miR-31 promoter contains p65 binding sites [51]. Furthermore, in their subsequent studies, it was demonstrated that miR-31 expression is upregulated in keratinocytes by NF- $\kappa \mathrm{B}$ signaling pathways activated by inflammatory cytokines [30]. However, another study described above confirmed that high expression of miR-31 can activate the NF- $\mathrm{kB}$ signaling pathway via inhibiting STK40 [49].

Although the two action pathways confirmed by these studies are different, both of them can promote keratinocyte proliferation and epidermal hyperplasia. Therefore, we hypothesize that a positive feedback loop may be formed by miR-31 and NF- $\kappa$ B signaling pathways in the hyperplastic keratinocytes (Fig. 2), which can be continuously aggravated by the development of psoriasis. If we want to intervene in the treatment of psoriasis through the miR-31/ NF- $\kappa \mathrm{B}$ signaling pathway, simultaneously blocking both pathways of the positive feedback loop may have doubling therapeutic effects.

But these studies mainly reflect the pathogenic role of miR-31 promoting hyperproliferation and abnormal differentiation of keratinocytes in psoriasis via the NF- $\kappa B$ signaling pathway, and the physiological role of miR-31 in keratinocytes has not been examined in this study. However, new research from others soon filled this gap. 
Wang et al.: Important Regulator of Keratinocytes: miR-31

\section{The RAS/MAPK Signaling Pathway}

The RAS/MAPK signaling pathway, which has been shown to play a critical role in skin wound healing, has been found to be activated by miR-31 in different tissues. RAS/MAPK pathway activation can mediate tissue epithelial regeneration and tumorigenesis [52-54]. The intriguing questions are whether miR-31 can activate the RAS/MAPK signaling pathway in keratinocytes and what happens when RAS/MAPK is activated. Experimental results of Shi et al. [30] gave us satisfactory answers.

Shi et al. [30] confirmed that high expression of miR-31 is primarily concentrated in the wound edge of keratinocytes. They observed that wound healing process was delayed in miR-31 cKO mice, caused by impaired keratinocyte proliferation and migration, and miR-31 cKO mice epidermal edge was shorter and thinner than in the control group. In order to investigate the effect insight of changes in miR-31 on the status of the RAS/MAPK signaling pathway, they examined some downstream components of the RAS/MAPK signaling pathway at the wound edge of miR-31 cKO mice, such as Cyclin D1, c-myc, and Slug. All of them were reduced in cKO mice wounds both at the protein and RNA levels. Meanwhile, experiments in vitro showed that the expression levels of c-Myc, Cyclin D1, and Slug in HaCaT cells treated with miR-31 mimics were significantly increased. In addition, U0126, as an extensively used inhibitor of RAS/MAPK, at least partly weakened the proliferation promotion effect of miR-31 mimics on keratinocytes. Finally, the direct targets of miR-31 were found to be the negative regulators of the RAS/MAPK signaling pathway, including Rasa1, Spred1, Spred2, and Spry4. They were confirmed to have miR-31 binding sites in their 3'-UTRs of transcripts encoding and were upregulated at both protein and RNA levels in the wound edge of miR-31 cKO mice [30]. Collectively, these findings demonstrate that miR-31 activates the RAS/MAPK signaling pathway by inhibiting negative regulators of the RAS/MAPK pathway, and its downstream components promote keratinocyte proliferation and migration.

\section{Epithelial Membrane Protein 1}

Li et al. [31] also found that miR-31 expression was upregulated in epidermal keratinocytes during skin wound healing. In order to determine the role of miR-31 in keratinocyte proliferation, they performed the CyQUANT cell proliferation test, which showed that the overexpression of miR-31 increased the number of cultured living cells, whereas the inhibition of miR-31 reduced the number of cultured living cells. Further studies showed that the increased expression of the proliferation marker Ki-67 in keratinocytes was changed synchronously by the expression of miR-31. The results of scratch assays showed that the overexpression of miR-31 significantly increased the migration ability of keratinocytes. Collectively, miR-31 is a positive regulator of keratinocyte growth and movement. To predict the potential targets of miR-31, three independent algorithms were used for comprehensive analysis: TargetScan, miRanda, and miRWalk. In this study, luciferase reporter assays and qRT-PCR showed that miR-31 directly negatively regulates epithelial membrane protein 1 (EMP-1) expression by binding to its predicted target in the $3^{\prime}$-UTR of mRNA. However, the function of EMP-1 in keratinocytes has been underexplored, so Li et al. [31] regulated the expression of EMP-1 in keratinocytes to observe its function. Notably, low EMP-1 expression led to a significant increase in Ki-67 expression and colony formation of keratinocytes, while the results of the scratch assays indicated that silencing EMP-1 increased the migration capacity of keratinocytes. These data suggest that EMP-1 is not only an important target for mediating the biological functions of miR-31 in keratinocytes, but also that silencing EMP-1 significantly enhances the proliferation and migration of keratinocytes by miR-31 [31]. Notably, Emp-1 
Wang et al.: Important Regulator of Keratinocytes: miR-31

has previously been shown to regulate the proliferation and migration of cancer cells in several different tissues [55-57]. But this is the first time that Emp-1 has been shown to have a positive effect on trauma recovery.

\section{Notch Signaling Pathway}

Keratinocytes are present not only in the skin, but also in the corneal epithelium, where their abnormal differentiation and hypoxia result in diabetic keratopathy [58]. Notch signaling has been proven to be one of the key determinants that orchestrate the proliferation and differentiation in epithelial keratinocytes. These signals are transmitted sequentially through different Notch ligands, receptors, and downstream pathways. Skin diseases such as psoriasis and basal cell, squamous cell carcinomas and diabetic keratopathy have been associated with abnormal Notch signaling, but how Notch signaling participated in these diseases is not well understood [59-61]. Peng et al. [61] found that FIH-1 expression was significantly increased in tissue from patients with psoriasis and corneal epithelium from patients with diabetic keratopathies. After further research, it was found that the expression of active markers Hes 1 and Hey 2 of the Notch signaling pathway will be reduced in FIH-1-transfected human epidermal keratinocytes. In addition, in the limbal epithelium cells from FIH-1 gene-deficient mice, it was observed that the expression levels of Hes 1 and Hey 2, keratinocyte differentiation marker PAI-2, and corneal epithelial differentiation marker mucin 1 (Muc1) increased; however, the expression of p63 and K15, which are undifferentiated corneal keratinocyte markers, decreased. These experimental results, while preliminary, suggest that FIH-1 negatively regulates the differentiation of keratinocytes by attenuating Notch signaling. miR-31 is an upstream regulatory gene of FIH-1, and miR-31 transfected human corneal epithelial cell lines showed reduced FIH-1 expression and increased PAI-2 and Muc1 expression.

As miR-31 is an upstream regulatory gene of FIH-1, overexpression of miR-31 was thought to enhance the proliferation and differentiation of keratinocytes. So, could miR-31 participate in the process of FIH-1/Notch signaling for regulating keratinocyte differentiation? As expected, lentiviral transduction of human corneal epithelial cell line with miR-31 reduced the expression of FIH-1, while expression of PAI-2 and Muc1 was increased. However, when Notch signaling in miR-31-transduced cells was inhibited by DAPT treatment, the expression of PAI-2 and Muc1 was also suppressed. These experiments illustrate that miR-31 can regulate the differentiation ability of keratinocytes through FIH-1/Notch signaling. Moreover, Peng et al. [61] emphasized that miR-31 is the key regulator miRNA for corneal epithelium proliferation through expression profiling analysis. The present results are significant in the application of miR-31/FIH-1/Notch signaling Nexus in the treatment of corneal epithelial lesions.

\section{Conclusions}

In view of the current and previous studies, the relationship between miR-31 and keratinocytes has gradually become clear. Almost all studies reveal that miR-31 is positively correlated with the proliferation, migration, and viability of keratinocytes, although it is accomplished through different target genes or signaling pathways. Given that skin is the largest non-specific immune organ, $\mathrm{NF}-\mathrm{kB}$, as a well-known immunoregulation signaling pathway, has become the most popular pathway to be studied [62]. As mentioned above, we indicate that a positive feedback loop may be formed by miR-31 and NF- $\mathrm{B}$, which can continuously enhance the proliferation, migration and activity of keratinocytes. Meanwhile, miR-31 
can promote keratinocyte proliferation and migration by activating the RAS/MAPK signaling pathway and its downstream components after being upregulated by the inflammatory signaling pathway [30]. In addition, as a direct target of miR-31, EMP-1 can enhance the capability of miR-31 to promote keratinocyte proliferation and migration, but the specific pathways of this process are unclear [31]. In corneal epithelium, miR-31 has been shown to regulate the differentiation capacity of keratinocytes through the FIH-1/Notch signaling pathway [61]. It is somewhat surprising that mi R-31 cKO mice do not significantly affect proliferation dynamics in the basal layer of the epidermis, whereas miR-31 mimics promote the proliferation and migration of keratinocytes around the wound edge. These results suggest that the loss of miR-31 does not affect the normal formation of skin tissue, but miR-31 can accelerate the healing of skin wounds. miR-31 is not essential for key physiological functions of epidermis under normal conditions [30]. miR-31 is more like an important regulator for keratinocytes, helping them rapidly proliferate, migrate, and dramatically increase cellular activity to complete the repair function needed by the body. Taken together, these findings help us understand what role miR-31 plays in key physiological functions, particularly in keratinocytes. And they provide a basis for the study of miR-31 in skin diseases.

Studies over the past decade have provided important information on functional differences in the injury response between epithelial cells from tissues from different locations, and the wound healing capacity of oral epithelial cells was much better than other epidermis $[63,64]$. In terms of embryology, oral cavity belongs to upper digestive tract organs in the digestive system, while oral mucosa exhibits exceptional healing capability that can heal wounds more quickly with reduced scar formation. These phenomena may be caused by the differences in physical environment and endogenous specific genes at particular anatomical sites [44]. Studies of the privileged healing response in oral mucosa demonstrated that the overexpression of microRNAome may underlie the ability of oral mucosal wounds to heal faster than skin wounds [45]. Furthermore, as the most stable and obvious miRNA from the overexpression microRNAome, miR-31 had been particularly concerned. It was confirmed that miR-31 was a key intrinsic element in the rapid healing response of oral mucosa in the subsequent vivo and vitro experiments [45]. However, given the existing experimental results, we do not know why the expression level of miR-31 in the oral mucosa is higher than other skin epithelium. Some results show that human saliva can stimulate migration of skin and oral mucosa fibroblasts and keratinocytes to accelerate wound closure and promote an innate inflammatory response (increased CCL20, IL-6, and CXCL-8 secretion) [65]. So, whether there are some special proteins and cytokines in the oral cavity or the digestive environment can induce the high expression of miR-31 to ensure the rapid completion of the wound healing process, this will be a very clinically significant study. When we find out why the high expression of miR-31 can be maintained in the oral mucosa, we can apply it to chronic gastric ulcers or other peptic ulcer diseases, as well as surgical operations. In postoperative care, the problem of wound infection could be effectively reduced because the speed of wound healing would be accelerated.

Overall, the overexpression of miR-31 can promote the proliferation, differentiation, and migration of keratinocytes, and further accelerate wound healing. These make miR-31 a potential therapeutic target for some pathological wound healing obstructive diseases such as chronic ulcers and delayed trauma closure. When miR-31 is downregulated, the proliferation, differentiation, migration, and activity of keratinocytes are inhibited; thus, miR-31 becomes a potential therapeutic target for some diseases concerned with abnormal keratinocyte proliferation and differentiation such as psoriasis and diabetic keratopathy. As a super-regulatory gene of keratinocytes, miR-31 should be a subject of more intense research with the hope of providing us new directions for treatment of immune skin diseases.

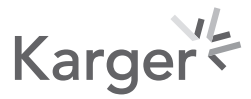




\section{Acknowledgement}

The authors would like to thank their teachers and students in their lab for their careful guidance and many useful suggestions regarding this article.

\section{Conflict of Interest Statement}

The authors have no conflicts of interest to declare.

\section{Funding Sources}

This work was funded by National Natural Science Foundation of China (NSFC, 81371384), Key Research and Development Program of Shanxi Province (201803D31068), Applied Basic Research Programs of Shanxi Province (201801D121212, 201901D111384), and Scientific and Technological Innovation Programs of Higher Education Institutions in Shanxi (2019L0418, 2019L0445).

\section{Author Contributions}

All authors contributed to the study conception and design. The first draft of the manuscript was written by Fei Wang, and all authors read and approved the final manuscript.

\section{References}

1 Koster MI, Roop DR. Mechanisms regulating epithelial stratification. Annu Rev Cell Dev Biol. 2007;23(1): 93-113.

2 Rousselle P, Braye F, Dayan G. Re-epithelialization of adult skin wounds: cellular mechanisms and therapeutic strategies. Adv Drug Deliv Rev. 2019 Jun;146:344-65.

3 Guo S, Dipietro LA. Factors affecting wound healing. J Dent Res. 2010 Mar;89(3):219-29.

4 Benhadou F, Mintoff D, Del Marmol V. Psoriasis: Keratinocytes or Immune Cells - Which Is the Trigger? Dermatology. 2019;235(2):91-100.

5 Albanesi C. Keratinocytes in allergic skin diseases. Curr Opin Allergy Clin Immunol. 2010 Oct;10(5):452-6.

6 Kutlubay Z, Engin B, Bairamov O, Tüzün Y. Acanthosis nigricans: A fold (intertriginous) dermatosis. Clin Dermatol. 2015 Jul-Aug;33(4):466-70.

7 Radeva MY, Walter E, Stach RA, Yazdi AS, Schlegel N, Sarig O, et al. ST18 Enhances PV-IgG-Induced Loss of Keratinocyte Cohesion in Parallel to Increased ERK Activation. Front Immunol. 2019 Apr; 10:770.

8 Gröne A. Keratinocytes and cytokines. Vet Immunol Immunopathol. 2002 Sep;88(1-2):1-12.

9 Sarra M, Caruso R, Cupi ML, Monteleone I, Stolfi C, Campione E, et al. IL-21 promotes skin recruitment of CD4(+) cells and drives IFN- $\gamma$-dependent epidermal hyperplasia. J Immunol. 2011 May;186(9):5435-42.

10 Piskin G, Sylva-Steenland RM, Bos JD, Teunissen MB. In vitro and in situ expression of IL-23 by keratinocytes in healthy skin and psoriasis lesions: enhanced expression in psoriatic skin. J Immunol. 2006 Feb;176(3): 1908-15.

11 Friedrich M, Tillack C, Wollenberg A, Schauber J, Brand S. IL-36y sustains a proinflammatory self-amplifying loop with IL-17C in anti-TNF-induced psoriasiform skin lesions of patients with Crohn's disease. Inflamm Bowel Dis. 2014 Nov;20(11):1891-901.

12 Lee RC, Feinbaum RL, Ambros V. The C. elegans heterochronic gene lin-4 encodes small RNAs with antisense complementarity to lin-14. Cell. 1993 Dec;75(5):843-54.

13 LaPierre MP, Stoffel M. MicroRNAs as stress regulators in pancreatic beta cells and diabetes. Mol Metab. 2017 Jul;6(9):1010-23.

14 Yonemori K, Kurahara H, Maemura K, Natsugoe S. MicroRNA in pancreatic cancer. J Hum Genet. 2017 Jan; 62(1):33-40.

15 Treiber T, Treiber N, Plessmann U, Harlander S, Daiß JL, Eichner N, et al. A Compendium of RNA-Binding Proteins that Regulate MicroRNA Biogenesis. Mol Cell. 2017 Apr;66(2):270-284.e13. 
16 Jinnin M. Recent progress in studies of miRNA and skin diseases. J Dermatol. 2015 Jun;42(6):551-8.

17 Meza-Sosa KF, Pérez-García EI, Camacho-Concha N, López-Gutiérrez O, Pedraza-Alva G, Pérez-Martínez L. MiR-7 promotes epithelial cell transformation by targeting the tumor suppressor KLF4. PLoS One. 2014; 9(9):e103987.

18 Giles KM, Brown RA, Ganda C, Podgorny MJ, Candy PA, Wintle LC, et al. microRNA-7-5p inhibits melanoma cell proliferation and metastasis by suppressing RelA/NF- $\kappa$ B. Oncotarget. 2016 May;7 (22):31663-80.

19 Yan H, Song K, Zhang G. MicroRNA-17-3p promotes keratinocyte cells growth and metastasis via targeting MYOT and regulating Notch1/NF-кB pathways. Pharmazie. 2017 Sep;72(9):543-9.

20 Jia HY, Zhang K, Lu WJ, Xu GW, Zhang JF, Tang ZL. LncRNA MEG3 influences the proliferation and apoptosis of psoriasis epidermal cells by targeting miR-21/caspase-8. BMC Mol Cell Biol. 2019 Oct;20(1):46.

21 Wang J, Qiu Y, Shi NW, Zhao JN, Wang YC, Jiang H, et al. microRNA-21 mediates the TGF- $\beta 1$-induced migration of keratinocytes via targeting PTEN. Eur Rev Med Pharmacol Sci. 2016 Sep;20(18):3748-59.

22 Lu X, Liu Y, Luo F, Zhang A, Liu X, Lu L, et al. MicroRNA-21 activation of Akt via PTEN is involved in the epithelial-mesenchymal transition and malignant transformation of human keratinocytes induced by arsenite. Toxicol Res (Camb). 2016 May;5(4):1140-7.

23 Li Q, Zhao H, Chen W, Huang P, Bi J. Human keratinocyte-derived microvesicle miRNA-21 promotes skin wound healing in diabetic rats through facilitating fibroblast function and angiogenesis. Int J Biochem Cell Biol. 2019 Sep;114:105570.

24 Jafarinejad-Farsangi S, Farazmand A, Gharibdoost F, Karimizadeh E, Noorbakhsh F, Faridani H, et al. Inhibition of MicroRNA-21 induces apoptosis in dermal fibroblasts of patients with systemic sclerosis. Int J Dermatol. 2016 Nov;55(11):1259-67.

25 Mao XH, Chen M, Wang Y, Cui PG, Liu SB, Xu ZY. MicroRNA-21 regulates the ERK/NF- $\kappa B$ signaling pathway to affect the proliferation, migration, and apoptosis of human melanoma A375 cells by targeting SPRY1, PDCD4, and PTEN. Mol Carcinog. 2017 Mar;56(3):886-94.

26 Jafarinejad-Farsangi S, Farazmand A, Mahmoudi M, Gharibdoost F, Karimizadeh E, Noorbakhsh F, et al. MicroRNA-29a induces apoptosis via increasing the Bax:Bcl-2 ratio in dermal fibroblasts of patients with systemic sclerosis. Autoimmunity. 2015;48(6):369-78.

27 Ciechomska M, O'Reilly S, Suwara M, Bogunia-Kubik K, van Laar JM. MiR-29a reduces TIMP-1 production by dermal fibroblasts via targeting TGF- $\beta$ activated kinase 1 binding protein 1 , implications for systemic sclerosis. PLoS One. 2014;9(12):e115596.

28 Yan S, Xu Z, Lou F, Zhang L, Ke F, Bai J, et al. NF- $\kappa B$-induced microRNA-31 promotes epidermal hyperplasia by repressing protein phosphatase 6 in psoriasis. Nat Commun. 2015 Jul;6(1):7652.

29 Abdallah F, Pichon C. Evidence on the direct correlation between miR-31 and IL-22 axis in IMQ-induced psoriasis. Exp Dermatol. 2019 Nov;28(11):1336-40.

30 Shi J, Ma X, Su Y, Song Y, Tian Y, Yuan S, et al. MiR-31 Mediates Inflammatory Signaling to Promote Re-Epithelialization during Skin Wound Healing. J Invest Dermatol. 2018 Oct;138(10):2253-63.

31 Li D, Li XI, Wang A, Meisgen F, Pivarcsi A, Sonkoly E, et al. MicroRNA-31 Promotes Skin Wound Healing by Enhancing Keratinocyte Proliferation and Migration. J Invest Dermatol. 2015 Jun;135(6):1676-85.

32 Luan L, Shi J, Yu Z, Andl T. The major miR-31 target genes STK40 and LATS2 and their implications in the regulation of keratinocyte growth and hair differentiation. Exp Dermatol. 2017 Jun;26(6):497-504.

33 Zheng Y, Sun Y, Liu Y, Zhang X, Li F, Li L, et al. The miR-31-SOX10 axis regulates tumor growth and chemotherapy resistance of melanoma via PI3K/AKT pathway. Biochem Biophys Res Commun. 2018 Sep;503(4): 2451-8.

34 Zhang G, Song K, Yan H. MicroRNA-124 represses wound healing by targeting SERP1 and inhibiting the Wnt/ $\beta$ catenin pathway. Adv Clin Exp Med. 2019 Jun;28(6):711-8.

35 Chang L, Liang J, Xia X, Chen X. miRNA-126 enhances viability, colony formation, and migration of keratinocytes HaCaT cells by regulating PI3 K/AKT signaling pathway. Cell Biol Int. 2019 Feb;43(2):182-91.

36 Xiong Y, Chen H, Liu L, Lu L, Wang Z, Tian F, et al. microRNA-130a Promotes Human Keratinocyte Viability and Migration and Inhibits Apoptosis Through Direct Regulation of STK40-Mediated NF- $\kappa$ B Pathway and Indirect Regulation of SOX9-Meditated JNK/MAPK Pathway: A Potential Role in Psoriasis. DNA Cell Biol. 2017 Mar; 36(3):219-26.

37 Feng SJ, Chu RQ, Ma J, Wang ZX, Zhang GJ, Yang XF, et al. MicroRNA138 regulates keratin 17 protein expression to affect HaCaT cell proliferation and apoptosis by targeting hTERT in psoriasis vulgaris. Biomed Pharmacother. 2017 Jan;85:169-76.

38 Shi K, Qiu X, Zheng W, Yan D, Peng W. MiR-203 regulates keloid fibroblast proliferation, invasion, and extracellular matrix expression by targeting EGR1 and FGF2. Biomed Pharmacother. 2018 Dec;108:1282-8.

39 Zhang Y, Zhang L, Zhang Q, Hong W, Lin X. [microRNA-222 regulates proliferation and apoptosis of fibroblasts in hypertrophic scar via matrix metalloproteinase 1]. Zhejiang Da Xue Xue Bao Yi Xue Ban. 2017 May;46(6): 609-17.

40 Kobayashi H, Tomari Y. RISC assembly: coordination between small RNAs and Argonaute proteins. Biochim Biophys Acta. 2016 Jan;1859(1):71-81.

41 O'Brien J, Hayder H, Zayed Y, Peng C. Overview of MicroRNA Biogenesis, Mechanisms of Actions, and Circulation. Front Endocrinol (Lausanne). 2018 Aug;9:402.

42 Zhang N, Hu G, Myers TG, Williamson PR. Protocols for the Analysis of microRNA Expression, Biogenesis, and Function in Immune Cells. Curr Protoc Immunol. 2019;126(1):e78. 
43 Chen L, Arbieva ZH, Guo S, Marucha PT, Mustoe TA, DiPietro LA. Positional differences in the wound transcriptome of skin and oral mucosa. BMC Genomics. 2010 Aug;11(1):471.

44 Simões A, Chen L, Chen Z, Zhao Y, Gao S, Marucha PT, et al. Differential microRNA profile underlies the divergent healing responses in skin and oral mucosal wounds. Sci Rep. 2019 May;9(1):7160.

45 Chen L, Simões A, Chen Z, Zhao Y, Wu X, Dai Y, et al. Overexpression of the Oral Mucosa-Specific microRNA-31 Promotes Skin Wound Closure. Int J Mol Sci. 2019 Jul;20(15):3679.

46 Henry TW, Mendoza FA, Jimenez SA. Role of microRNA in the pathogenesis of systemic sclerosis tissue fibrosis and vasculopathy. Autoimmun Rev. 2019 Nov;18(11):102396.

47 Ichihara A, Jinnin M, Yamane K, Fujisawa A, Sakai K, Masuguchi S, et al. microRNA-mediated keratinocyte hyperproliferation in psoriasis vulgaris. Br J Dermatol. 2011 Nov;165(5):1003-10.

48 Lerman G, Avivi C, Mardoukh C, Barzilai A, Tessone A, Gradus B, et al. MiRNA expression in psoriatic skin: reciprocal regulation of hsa-miR-99a and IGF-1R. PLoS One. 2011;6(6):e20916.

49 Xu N, Meisgen F, Butler LM, Han G, Wang XJ, Söderberg-Nauclér C, et al. MicroRNA-31 is overexpressed in psoriasis and modulates inflammatory cytokine and chemokine production in keratinocytes via targeting serine/threonine kinase 40. J Immunol. 2013 Jan;190(2):678-88.

50 Goldminz AM, Au SC, Kim N, Gottlieb AB, Lizzul PF. NF- $\kappa B$ : an essential transcription factor in psoriasis. J Dermatol Sci. 2013 Feb;69(2):89-94.

51 Tian Y, Ma X, Lv C, Sheng X, Li X, Zhao R, et al. Stress responsive miR-31 is a major modulator of mouse intestinal stem cells during regeneration and tumorigenesis. eLife. 2017 Sep;6:e29538.

52 Li X, Li D, Wikstrom JD, Pivarcsi A, Sonkoly E, Ståhle M, et al. MicroRNA-132 promotes fibroblast migration via regulating RAS p21 protein activator 1 in skin wound healing. Sci Rep. 2017 Aug;7(1):7797.

53 Edmonds MD, Boyd KL, Moyo T, Mitra R, Duszynski R, Arrate MP, et al. MicroRNA-31 initiates lung tumorigenesis and promotes mutant KRAS-driven lung cancer. J Clin Invest. 2016 Jan;126(1):349-64.

54 Sun D, Yu F, Ma Y, Zhao R, Chen X, Zhu J, et al. MicroRNA-31 activates the RAS pathway and functions as an oncogenic MicroRNA in human colorectal cancer by repressing RAS p21 GTPase activating protein 1 (RASA1). J Biol Chem. 2013 Mar;288(13):9508-18.

55 Lai S, Wang G, Cao X, Li Z, Hu J, Wang J. EMP-1 promotes tumorigenesis of NSCLC through PI3K/AKT pathway. J Huazhong Univ Sci Technolog Med Sci. 2012 Dec;32(6):834-8.

56 Liu C, Wei X, Li F, Wang L, Ruan X, Jia J, et al. The Prognostic Value of Epithelial Membrane Protein 1 (EMP-1) in Patients with Laryngeal Carcinoma. Med Sci Monit. 2017 Aug;23:3795-800.

57 Sun G, Zhao G, Lu Y, Wang Y, Yang C. Association of EMP1 with gastric carcinoma invasion, survival and prognosis. Int J Oncol. 2014 Sep;45(3):1091-8.

58 Peng H, Hamanaka RB, Katsnelson J, Hao LL, Yang W, Chandel NS, et al. MicroRNA-31 targets FIH-1 to positively regulate corneal epithelial glycogen metabolism. FASEB J. 2012 Aug;26(8):3140-7.

59 Massi D, Panelos J. Notch signaling and the developing skin epidermis. Adv Exp Med Biol. 2012;727:131-41.

60 Dziunycz PJ, Neu J, Lefort K, Djerbi N, Freiberger SN, Iotzova-Weiss G, et al. CYFIP1 is directly controlled by NOTCH1 and down-regulated in cutaneous squamous cell carcinoma. PLoS One. 2017 Apr;12(4):e0173000.

61 Peng H, Kaplan N, Hamanaka RB, Katsnelson J, Blatt H, Yang W, et al. microRNA-31/factor-inhibiting hypoxiainducible factor 1 nexus regulates keratinocyte differentiation. Proc Natl Acad Sci USA. 2012 Aug;109(35): 14030-4.

62 Collignon E, Canale A, Al Wardi C, Bizet M, Calonne E, Dedeurwaerder S, et al. Immunity drives TET1 regulation in cancer through NF-кB. Sci Adv. 2018 Jun;4(6):eaap7309.

63 Iglesias-Bartolome R, Uchiyama A, Molinolo AA, Abusleme L, Brooks SR, Callejas-Valera JL, et al. Transcriptional signature primes humanoral mucosa for rapid wound healing. SciTransl Med.2018Jul;10(451):eaap8798.

64 Wong JW, Gallant-Behm C, Wiebe C, Mak K, Hart DA, Larjava H, et al. Wound healing in oral mucosa results in reduced scar formation as compared with skin: evidence from the red Duroc pig model and humans. Wound Repair Regen. 2009 Sep-Oct;17(5):717-29.

65 Rodrigues Neves C, Buskermolen J, Roffel S, Waaijman T, Thon M, Veerman E, et al. Human saliva stimulates skin and oral wound healing in vitro. J Tissue Eng Regen Med. 2019 Jun;13(6):1079-92. 